\title{
Actions entreprises à Marseille en vue de protéger les zones de baignade contre les pollutions liquides
}

\author{
Jean Marc Sotty $\left({ }^{*}\right)$ Jean Paul Charry $\left({ }^{*}\right)$ Yves Sanchez $(* *)$ \\ (*) Service de l'Assainissement - Ville de Marseille - 38/40 Quai de Rive Neuve - 13007 Marseille \\ (**) SERAM (Société d'Exploitation du Réseau d'Assainissement de Marseille) - 24a Rue Fort Notre Dame \\ - 13007 Marseille
}

\section{Introduction}

Pour toutes les villes littorales, la qualité des eaux de mer est un facteur déterminant pour de multiples aspects de leur vie quotidienne: activités balnéaires, portuaires, pêche. Il est souvent considéré que la construction d'une station d'épuration ayant pour vocation de traiter les eaux usées domestiques auxquelles s'ajoutent fréquemment des eaux industrielles est la solution pour résoudre l'essentiel des problèmes du milieu récepteur. S'il est vrai que le rôle de ces unités d'épuration est déterminant au regard d'un examen global de la situation, son impact peut s'avérer localement insuffisant par suite d'arrivées permanentes ou accidentelles d'eaux polluées à même le littoral même si elles peuvent être a priori estimées comme peu importantes.

Les pollutions locales précitées peuvent avoir de multiples origines :

i) Rejets d'eaux usées non collectées par le réseau d'assainissement.

ii) Rejets en provenance des ouvrages pluviaux liés à des raccordements anormaux d'eaux usées et au rejet des eaux de lavage des chaussées.

iii) Trop plein de stations de pompage en cas de dysfonctionnement.

iv) Déversoirs de réseaux unitaires par temps de pluie.

Il convient de signaler qu'à Marseille les plages les plus fréquentées sont localisées en zone urbaine.

La connaissance de l'importance relative de ces différentes sources de pollution est nécessaire. Afin que la population par des réactions trop hâtives, ne mette en doute l'efficacité et l'intérêt des stations d'épuration qui ont été construites au prix d'efforts économiques importants, des mesures techniques se justifient.

L'objet de la présente communication est de rappeler l'importance relative de la pollution apportée par les eaux usées, les eaux pluviales, les eaux de nettoyage de la voirie et de présenter les dispositifs mis en œuvre pour maîtriser la pollution littorale en complément de l'épuration des eaux usées.

\section{Les sources de pollution}

Elles peuvent être classées de la façon suivante :

1.1. Rejets directs en mer d'effluents sanitaires d'habitations côtières, de cabanons occupés essentiellement l'été ou d'habitations situées en bord de mer et en contrebas des réseaux d'assainissement.

\subsection{Rejets en provenance des ouvrages pluviaux}

Sur les zones à vocation balnéaire (assainies en système séparatif) les collecteurs pluviaux ont leurs exutoires sur les plages. Ces ouvrages peuvent générer une pollution importante par temps de pluie mais également par temps sec du fait du raccordement anormaux d'eaux usées ou d'apports d'eaux de lavage de Voirie.

Il convient de noter que les eaux de lavage des chaussées engendrent une pollution importante tant du point de vue bactériologique que physico chimique.

Les études faites à Marseille ont montré que :

- Les eaux de voirie amènent dans le milieu naturel un effluent fortement pollué pendant un temps très court $(2$ à $5^{\prime}$ maximum) lorsque les ouvrages concernés présentent une longueur inférieure à $500 \mathrm{~m}$.

- Les concentrations observées sont 4 à 5 fois plus élevées que celle d'une eau usée domestique.

- Une décantation de deux heures permet une amélioration très nette de la qualité des eaux. Nous obtenons une réduction de $77 \%$ de la DCO de de $80 \%$ des MES.

- Les teneurs en plomb peuvent atteindre 14 à 16 fois celles d'une eau usée domestique.

- La contamination bactérienne est toujours très importante, $10^{11}$ à $10^{12}$ coliformes $/ 100 \mathrm{ml}$.

La diminution des paramètres bactériologiques est beaucoup plus lente que celle des paramètres physicochimiques. Après une heure d'écoulement, l'effluent est toujours pollué, $10^{4}$ à $10^{5}$ coliformes $/ 100 \mathrm{ml}$.

Les résultats de ces études sont consignés dans les tableaux et graphes donnés en annexe $n^{\circ} 1$. 


\subsection{Incidents de fonctionnement des stations de pompage d'eaux usées}

L'assainissement du littoral à vocation balnéaire marseillais est tributaire de 50 stations de relevage qui acheminent les eaux usées de ces zones vers la station d'épuration.

Ces équipements électromécaniques sont gérés localement par de petits automatismes câblés asservissant les pompes aux débits entrants.

Initialement l'exploitation de ces sites consistait à faire des visites quotidiennes d'entretien et de vérifications préventives.

Toute anomalie de fonctionnement survenant en dehors de ces visites n'était perceptible que de l'extérieur, au moment où apparaissaient les nuisances sur le milieu récepteur (déversement dû à un arrêt des pompes).

Les interventions correctives se faisaient souvent avec un retard important par rapport au début de l'évènement, et entraînaient ainsi des nuisances accrues.

\subsection{Rejets en provenance des déversoirs d'orage}

Le centre ville de Marseille étant désservi par un réseau de type unitaire, l'un des déversoirs d'orage de ce dernier peut apporter des pollutions par temps de pluie.

\section{Actions entreprises à Marseille pour protéger les plages}

\subsection{Suppression des rejets illicites d'eaux usées sur le litto- ral}

Supprimer des rejets diffus d'eaux usées qui peuvent aboutir sur le littoral peut paraître secondaire dans la mesure où les charges polluantes (organiques ou particulaires) ne sont pas importantes. Même dans ce cas, compte tenu de l'importance des aspects bactériologiques pour les eaux de baignade, cette action est indispensable.

Des enquêtes longues et difficiles nécessitant un personnel fiable, connaissant bien le réseau, ont dû être menées. Il s'agit de recenser puis de faire supprimer tout rejet d'eaux usées (ne serait-ce que ceux d'une seule habitation). Les méthodes d'exploration par traçage chimique, inspection par caméra vidéo, fumigènes ont été utilisées.

La contribution d'un laboratoire d'analyse chimique et bactériologique est aussi nécessaire.

La méthodologie adoptée à Marseille a été la suivante : i) Inventaire de tous les exutoires en mer à partir de plans du réseau mais aussi par prospection systématique sur le terrain,

ii) Détermination des priorités d'études à partir de mesures de pollution réalisées aux exutoires en mer.

iii) Repérage - recensement et caractérisation des rejets anormaux,

iv) Mise en conformité des branchements par voie administrative.
Compte tenu de l'importance du problème et des difficultés de réalisation, l'aboutissement d'un tel programme nécessite plusieurs années de travail.

\subsection{Suppression des rejets sanitaires dans les ouvrages pluviaux et captage des eaux de lavage des voiries dans les réseaux unitaires}

La suppression des rejets sanitaires dans les ouvrages pluviaux se fait par mise en œuvre de la méthodologie décrite au paragraphe 2.1. S'agissant du problème des eaux de lavage des voiries, le nettoyage à sec des rues ne pouvant être envisagé, car mal accepté par la population, un palliatif a du être recherché avec pour objectif d'avoir, autant que faire se peut, des débits nuls aux exutoires des ouvrages pluviaux et ruisseaux situés sur la zone des plages.

La solution adoptée à Marseille (cf schémas $n^{\circ} 1$ et 2 donnés en annexe 2) consiste à dévier dans le réseau sanitaire les eaux de lavage de la voirie ainsi que les premiers flots d'eaux pluviales. Ces eaux sont ainsi acheminées vers la station d'épuration pour y être traitées.

A contrario, il est important de ne pas surcharger sur le plan hydraulique le réseau sanitaire et de ne pas entraîner une dilution des effluents par rapports d'eaux propres.

Pratiquement, il a été créé une liaison gravitaire entre ouvrage pluvial et ouvrage sanitaire le plus proche.

Pour que l'ouvrage pluvial puisse, lors de fortes précipitations, reprendre sa vocation première (comme indiqué dans le schéma $n^{\circ} 2$ ) il a été installé sur chaque canalisation de by-pass EP/EU un clapet qui a été ensuite remplacé par des vannes motorisées.

Ces vannes sont commandées localement en tout ou rien par un système d'électrodes de détection de niveau.

Ce dispositif est relié au Réseau informatique centralisé, ce qui permet de connaître à tout moment la position de la vanne et éventuellement de procéder à une télécommande à distance (ouverture - fermeture).

La liaison "vanne by-pass"- réseau informatique centralisé se fait sur réseau autocommuté, soit par prise de ligne de l'unité locale qui envoie un message au PC en cas d'anomalie sur le site, soit par interrogation automatique cyclique du Réseau informatique centralisé vers l'unité locale une fois pas jour, soit par interrogation provoquée par l'opérateur de quart du PC.

Dans le plan global de gestion automatisée des réseaux d'assainissement, il est prévu qu'un logiciel de décision, intégrant les données pluviométriques, gèrera la fermeture des vannes de façon séquentielle afin de ne pas mettre en charge le réseau sanitaire. Ceci sous-entend, au préalable, une étude fine des capacités hydrauliques des réseaux.

Pour que les dispositifs donnent leur pleine efficacité, il a été nécessaire de mettre en œuvre des mesures d'accompagnement: sensibilisation des agents du Service du nettoiement et de la propreté sur la nécessité de limiter les débits d'eau de lavage des chaussées et de procéder à l'élimination par voie sèche des déchets solides souillant la voie publique.

Une telle évolution des habitudes ne se fait pas sans difficulté. 


\subsection{Télésurveillance des stations de pompage}

Les stations de relevage ont été équipées d'unités électroniques de traitement qui assurent les fonctions suivantes:

a - récupération des informations sur l'état de marche de la station:

- marche/arrêt

- défaut

- coupure EDF

- niveau de déversement..

b - émission de ces signaux vers le poste central, sur réseau P\&T autocommuté lorsque apparaît un évènement de type défaut,

c - réponses aux appels du PC,

d - établissement de comptages journaliers sur le nombre d'heures de fonctionnement et sur la consommation énergétique de chaque pompe

Communication au PC lors d'appels périodiques automatisés.

La surveillance permanente ainsi assurée permet une exploitation plus rationnelle de ces installations.

Les informations d'anomalies arrivant sur le PC sont mémorisées et répercutées immédiatement, par radio ou téléphone, à des équipes d'interventions rapides véhiculées réduisant considérablement le temps de réponse des réparations.

Ces données archivées permettent d'établir des bilans d'exploitations sur le fonctionnement de ces stations et ainsi d'adapter nos actions en fonction du comportement de chacune d'elles.

\section{Résultats}

Annuellement, environ 650 défauts sont détectés par le réseau informatique centralisé sur la soixantaine de stations de pompage et vannes by-pass mises en place sur le littoral.

Grâce à la télésurveillance et aux moyens d'intervention rapides mis en place, ce nombre d'anomalies, dont certaines présenteraient des risques potentiels importants de pollution pour le milieu récepteur, n'a conduit que dans $7 \%$ des cas à des déversements effectifs qui n'ont d'ailleurs très souvent pas excédé la dizaine de minutes.

\subsection{Minimisation des rejets en provenance des déversoirs d'orage}

Le principal déversoir du "Grand émissaire " (collecteur drainant les eaux du réseau unitaire) a comme exécutoire le Parc balnéaire du PRADO. Sa capacité de déversement est de $8 \mathrm{~m}^{3} / \mathrm{s}$.

En fonctionnement statique (seuil maçonné fixe) nous étions amenés à constater 12 à 15 déversements par an sur les plages lors d'épisodes pluvieux.
L'impossibilité de réglage de ce dispositif entraînait, en période de fortes pluies des risques d'inondation et en période de faibles pluies des risques de pollution.

Ce constat nous a conduit à mettre au point un système d'ouvrages mobiles permettant au réseau de contenir l'effluent jusqu'à la limite inférieure du risque d'inondation.

Notre choix s'est porté sur une solution consistant à commander le déversement au moyen de clapets métalliques assujettis à des verins hydrauliques horizontaux.

\section{Principe de fonctionnement}

En période de veille les clapets sont ouverts. Il est procédé à l'analyse permanente du niveau dans l'émissaire en amont et en aval du déversoir, par des capteurs ultrasoniques et piézo résistifs avec temporisation de la mesure et suppression de mesures aberrantes.

Lorsqu'en cas de pluie ou pour toute autre raison, le niveau de l'eau dans l'émissaire monte, la commande de fermeture des clapets a lieu dans tous les cas, avant que l'eau ne passe dans le déversoir. Les clapets sont donc, toujours fermés sans effort contrariant cette fermeture.

Lorsque l'émissaire se met en charge jusqu'à un niveau inacceptable, l'ouverture des clapets est commandée : par groupe de un, deux, trois ou quatre clapets.

Ce fonctionnement se fait aussi bien :

- en commande manuelle à partir de l'armoire électrique de la chambre de commande,

- en commande automatique à partir de l'automate programmable local,

- en télécommande depuis le PC informatique qui supervise en permanence le site.

\section{Perspectives et résultats}

La construction de cet ouvrage mobile s'intègre dans un vaste programme d'études et de réalisations portant sur :

- la connaissance hydraulique des réseaux par la connaissance hydro-pluviométrique du bassin unitaire (25 stations)

- le diagnostic du fonctionnement par la modélisation (modèles mathématiques RERAM et CAREDAS).

- la mise en œuvre de capacités de rétention des eaux permettant un traitement maximum par la station d'épuration ainsi que le réaménagement de certains points du réseau trop complexe et l'implantation d'autres ouvrages mobiles,

- la gestion automatisée et centralisée de l'ensemble cidessus. 
L'équipement du déversoir du PRADO qui concerne les zones de baignade et qui constitue une des premières étapes de ce programme visant à la maîtrise du réseau d'assai- nissement avant l'an 2000 a permis d'abaisser la fréquence de déversement sur les plages de 12 à 15 déversements/an à 1 à 3 déversements/an.

\section{Conclusion}

Les eaux usées d'une agglomération qui sont collectées, souvent traitées avant rejet en un point déterminé du littoral, ne sont pas la seule source de pollution. Les eaux pluviales ainsi que les eaux de lavage de la voirie apportent localement sur le littoral une pollution non négligeable surtout sur le plan bactériologique.

Il est important de maîtriser ces sources de pollution afin de garantir la qualité des eaux de baignade. Les actions engagées à Marseille pour atteindre cet objectif sont les suivantes :

- suppression des rejets illicites d'eaux usées dans les ouvrages pluviaux,

- déviation dans le raiseau sanitaire des eaux de lavage de la voirie et des premiers flux d'eaux pluviales,

- mise en place d'un système de gestion informatisée pour la centralisation des informations et des commandes d'organes de régulation du réseau,

- mise en place d'un système de déversoirs mobiles sur le réseau unitaire.

L'ensemble de ces mesures qui doit faire l'objet d'un programme général à traiter en parallèle avec l'épuration des eaux usées permet de donner à l'assainissement des agglomérations littorales toute l'efficacité attendue.

Les résultats donnés en annexe 3, obtenus à Marseille sur la qualité des eaux de baignade, confirment tout l'intérêt d'un tel programme.
Annexe 1

POLLUTION DES EAUX DE LAVAGE DES CHAUSSÉES

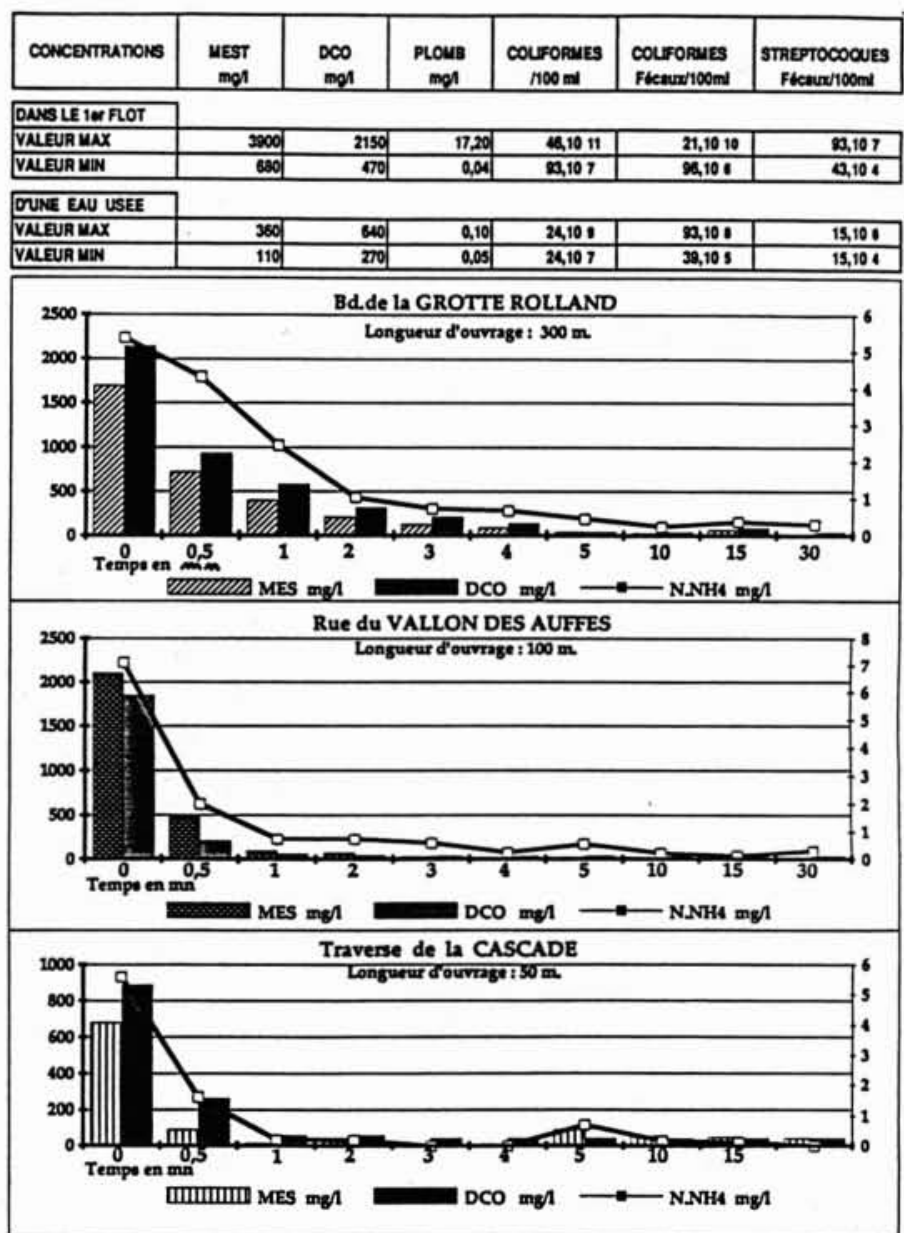




\section{Annexe 2}

FONCTIONNEMENT DES OUVRAGES DE BYPASS

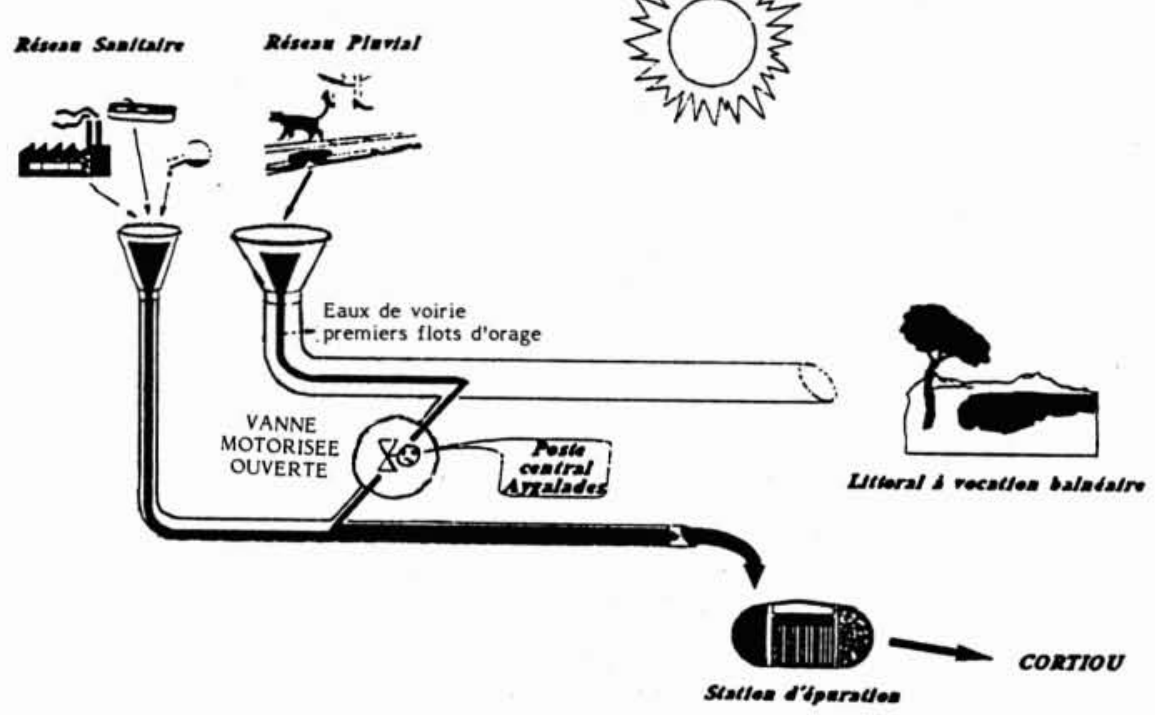

par temps sec ou lors d'épisodes pluvieux de faible intensité

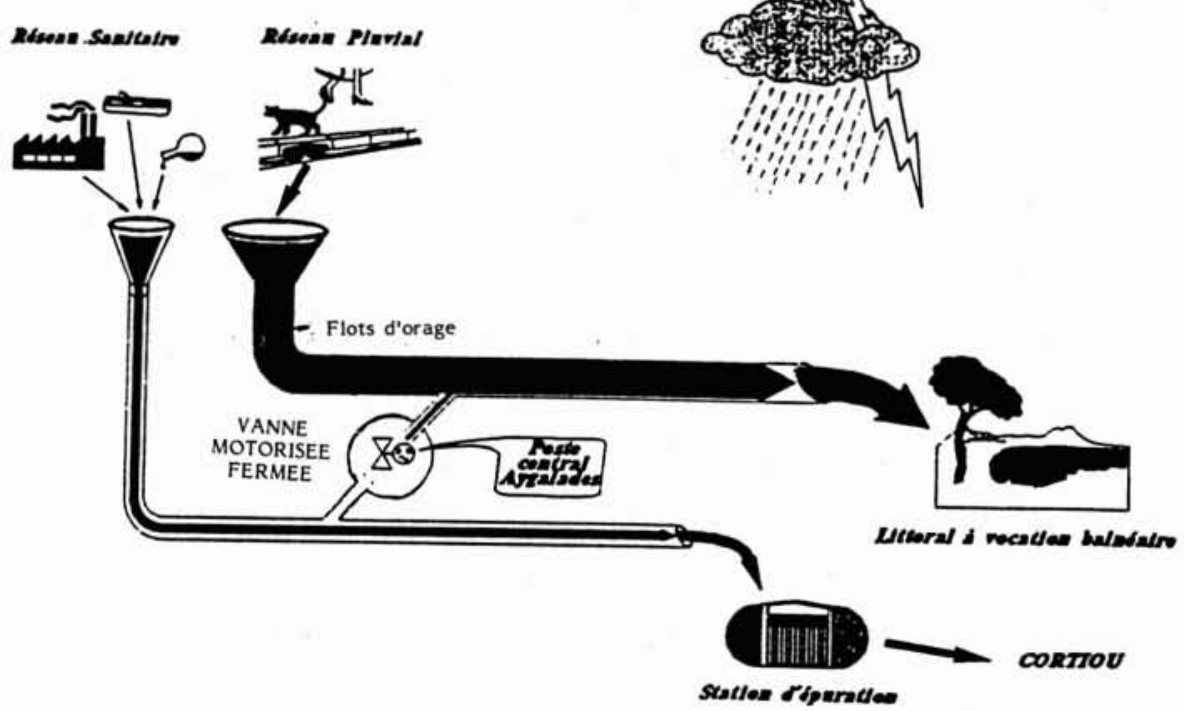

lors d'épisodes pluvieux de forte intensité 
Annexe 3

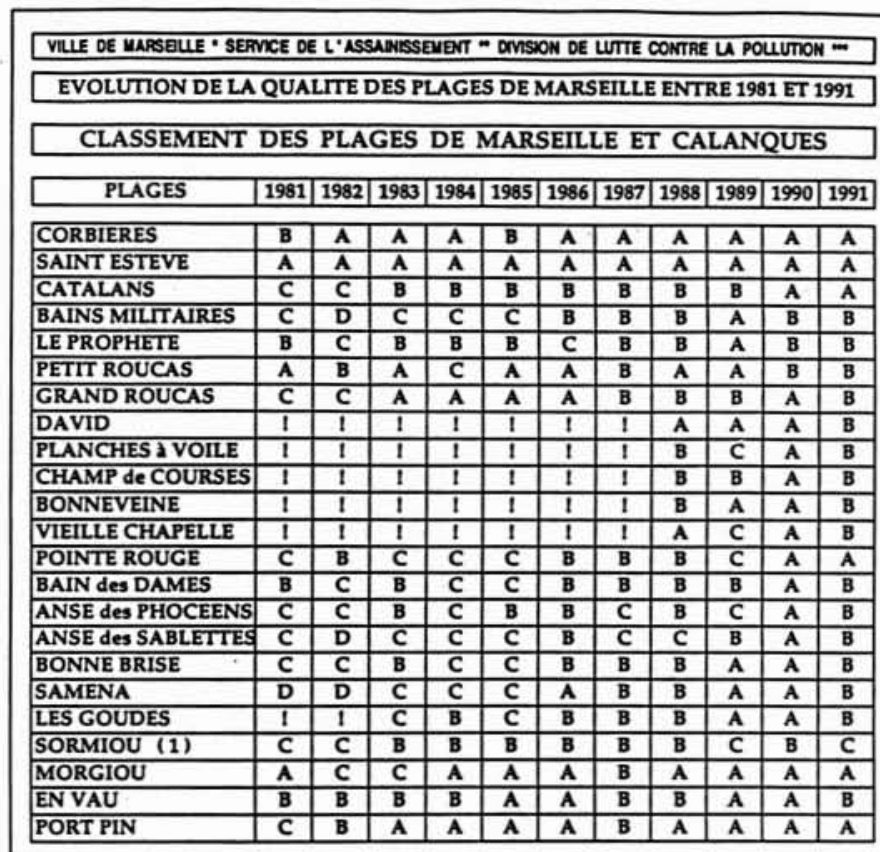

QUALITE DES EAUX DES PLAGES

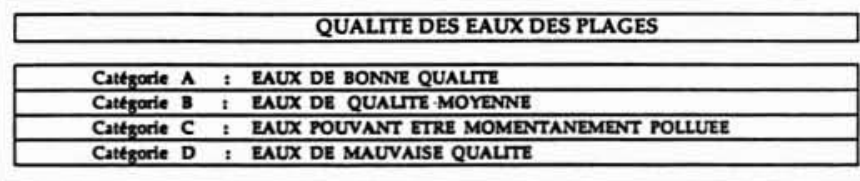

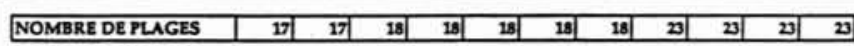

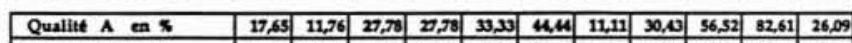

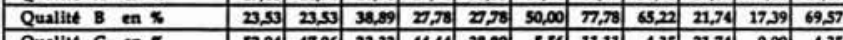

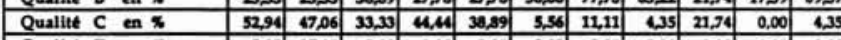

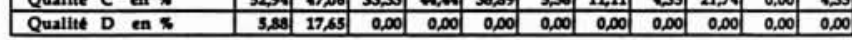

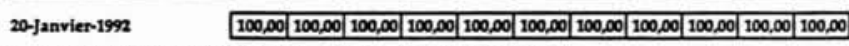

(1) 4 CUUMOUE OE SORLOU W'EST PAS STUEE ONS UNE ZONE ASSWNEE.

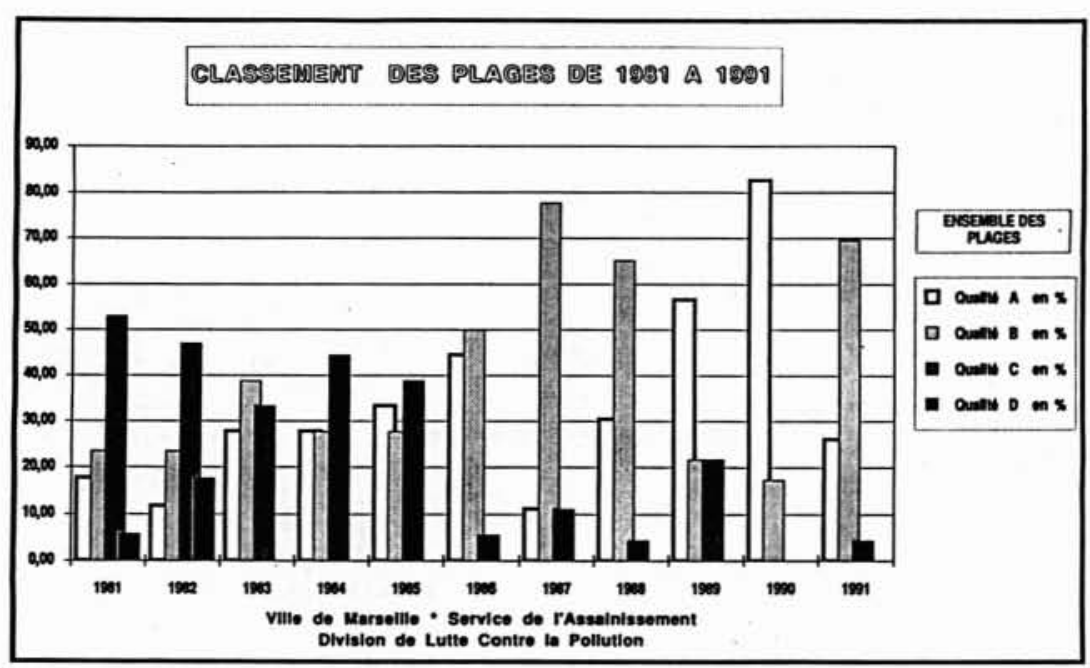

